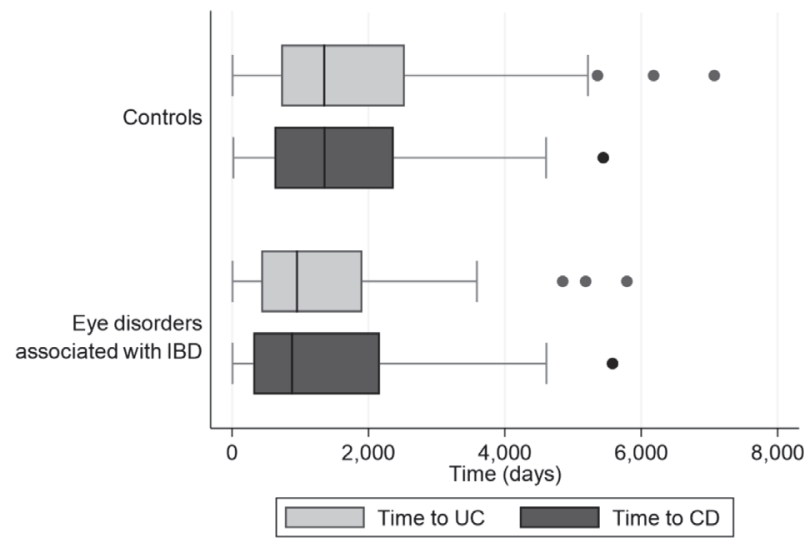

Abstract P121 Figure 1 Time to IBD diagnosis

diagnosis of IBD in cases and controls was compared using the Mann-Whitney U test.

Results 36883 subjects (median age 50 (IQR 37-65), 58\% female) with a new diagnosis of an eye disorder associated with IBD were matched to 102622 controls between 19952018. Uveitis made up 57\% of eye disorder cases. 196 $(0.53 \%)$ IBD cases were diagnosed in eye disorder subjects and $223(0.02 \%)$ in controls. Median time to UC diagnosis was 952 days for subjects with eye disorders and 1351 for controls, $\mathrm{p}=0.170$. Median time to $\mathrm{CD}$ diagnosis was 879 days and 1356 in controls, $\mathrm{p}=0.013$. Overall, median time to IBD diagnosis was 905 days compared to 1386 in controls, $\mathrm{p}<0.001$.

Figure 1 is a boxplot of time to ulcerative colitis (UC) and Crohn's disease (CD) diagnosis for eye disorder subjects compared to controls.

The rate of UC diagnoses in eye disorders was 70\% higher than in controls, IRR 1.73 (95\%CI 1.32-2.27). The rate of $\mathrm{CD}$ diagnoses was more than 3 -fold higher in the eye disorder group, 3.55 (2.68-4.71). Overall, eye disorders had a greater than 2 -fold rate of IBD diagnoses compared to controls, 2.44 (2.01-2.96). Eye disorder subjects had a $0.53 \%$ excess risk of IBD when compared to controls. When subjects coded for loperamide use, diarrhoea, anaemia, weight loss or lower gastrointestinal bleed within 6-months of study start were examined, eye disorder cases had a $1.6 \%$ excess IBD risk compared to controls.

Conclusions Eye disorders associated with IBD are commonly seen in isolation and health care professionals caring for those with these conditions may not consider IBD, leading to diagnostic delay. The relative risk of later IBD is high in such eye disorders and symptoms suggestive of IBD should be sought and screening investigations such as faecal calprotectin and gastroenterology referral considered.

\section{P122 SWITCHING FROM ORIGINATOR ADALIMUMAB TO BIOSIMILAR SB5(IMRALDI) - IBD SERVICE ASSESSMENT AND NEEDS}

Ioannis Koumoutsos, Susan Cowperthwaite, Sharon Glover, Chloe Sheil, Vithushan Vakeeswarasarma*, Christopher Strickland. Southend Hospital, Essex, UK

10.1136/gutjnl-2020-bsgcampus. 197

Introduction Biosimilar versions of adalimumab became available in the UK in late 2018. BSG suggests that automatic substitution to biosimilars would be inappropriate and patients should be switched to biosimilar if stable or in remission.

Aim To review whether biosimilar of Adalimumab(SB5) was inferior to originator drug and assess efficiency of IBD service throughout the process.

Methods We reviewed Adalimumab prescriptions from 1/2015 until 12/2018 when the switch to biosimilars was performed. Disease activity was assessed using laboratory parameters (Creactive protein (CRP) and faecal calprotectin (FC) where available and patient reported outcomes.

Results In total 121 Adalimumab prescriptions were issued from 1/2015 until 12/2018. We identified 77 patients that were switched to SB5 Adalimumab. Despite having an automatic substitution being implemented by our pharmacists, none of the patients declined change of treatment due to associated cost savings and only one patient requested to return to the originator following clinical deterioration.

Secondary loss of response 52 wks post switch of treatment occurred in $16.8 \%(13 / 77)$ following change to biosimilar, whereas $12.4 \%(15 / 121)$ patients have experienced secondary loss of response to originator drug prior to transition period (p:0.3). 23.3\% of patients reported clinical deterioration of symptoms, and $13 \%(10 / 77)$ of pts were changed to second line biosimilar due to side effects (mainly pain at the site of injection).

From patients with baseline biochemical markers available, $25.3 \%(18 / 71)$ of patients had raised CRP and 36\% (17/45) of patients had raised calprotectin. Worsening of CRP and faecal calprotectin were noted in $33 \%$ and $43 \%$ of these respectively. Subtherapeutic Adalimumab levels $(<5 \mathrm{UG} / \mathrm{ml})$ were identified in 14 patients but non-significant change of Adalimumab levels was seen in patients that had levels performed prior and after the transition period.

In terms of follow up, $28.6 \%(22 / 77)$ of patients were not seen 6 months pre or post transition period, and $35 \%$ (27/77) of patients still have not been reviewed 1 year post transition.

Conclusion Biosimilar SB5 was not inferior to originator and patient acceptance was very good due to associated cost savings. Whereas switching to a biosimilar should be performed in patients in remission, $25-36 \%$ had biochemical markers suggestive of active disease. Follow up of patients was suboptimal due to staffing issues. This indicates the importance of investment of cost savings back into IBD services to optimise their performance.

\section{P123 ARE WE ADDRESSING THE TOP TEN RESEARCH PRIORITIES IN MANAGEMENT OF IBD IN THE UK?}

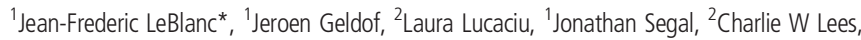
${ }^{1}$ Ailsa L Hart. 'St. Mark's Hospital, Harrow, UK; ${ }^{2}$ Western General Hospital, Edinburgh, UK

\subsection{6/gutjnl-2020-bsgcampus.198}

Introduction Three years after publication of the top 10 research priorities in Inflammatory Bowel Disease (IBD) based on the James Lind Alliance (JLA) Priority Setting Partnership, the question remains whether this initiative has influenced the research landscape. ${ }^{1}$ Therefore, the aim of this study is to create an overview of the current distribution of the research interests of clinical trials, in adults with IBD, ongoing or completed in the United Kingdom (UK) within the last 3 years. 


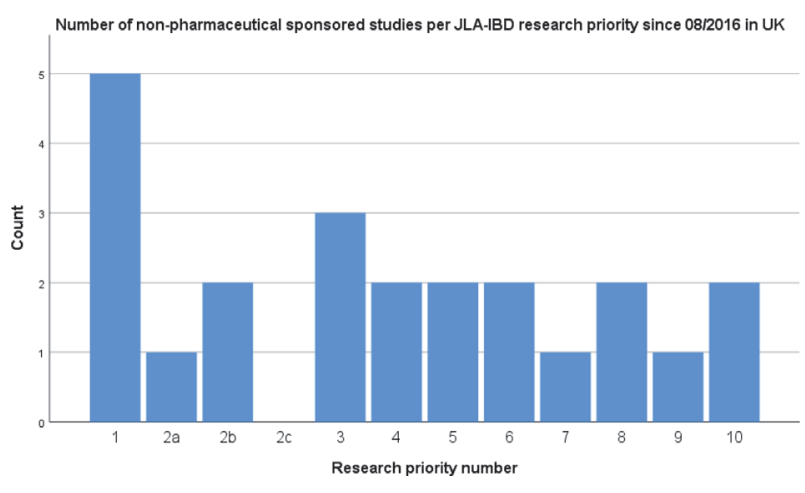

Abstract P123 Figure 1

Methods The database from Clinicaltrials.gov and European Union Clinical Trials Register was used to generate a list of all clinical studies set up in the UK involving adult patients with IBD. Trials posted on 09/08/2016 (publication date of the top 10 research questions) up to $16 / 11 / 2019$ were included. The register was completed by trials found to be relevant based on author consensus.

Results Twenty-one non-pharmaceutical-funded clinical studies in the UK were found of which eight were randomised clinical trials, 6 were non-randomised (open label) and 7 were observational studies. As shown in figure 1, 24\% of the nonpharmaceutical-driven IBD studies investigated the optimal treatment strategy considering efficacy, safety and cost-effectiveness (immunomodulators, biologics, surgery) in IBD (Priority 1). Four studies evaluated the role of diet in IBD management (Priorities 3 and 7). Development or assessment of biomarkers for stratification of patients (Priority 2) was subject of 3 studies. Fatigue, IBD-related pain, control of diarrhoea and incontinence were each subject of 2 studies (respectively Priorities 4, 8 and 6). Measuring the effect of altered gut microbiota (Priority 10) and determining the optimal treatment strategy for perianal Crohn's disease (Priority 5) was the target of 2 studies each. One study assessed surgical treatment for terminal ileal Crohn's disease (Priority 9).

Conclusions Optimal treatment strategy and the role of dietary interventions in IBD have been the most commonly studied domains in UK-led trials not sponsored by industry over a three year-period, accounting respectively for $24 \%$ and $19 \%$ of all studies. However, only 7 studies focused on patientreported outcomes such as IBD-related pain, fatigue, and diarrhoea/incontinence. As a consequence, it remains debatable to what extent the current landscape of clinical trials adequately represents the patients' viewpoint on needs for expanded knowledge in IBD.

\section{REFERENCE}

1. Hart AL, Lomer M, et al. (2017) What Are the Top 10 Research Questions in the Treatment of Inflammatory Bowel Disease? A Priority Setting Partnership with the James Lind Alliance. J Crohns Colitis 11, 204-211.

\section{P124 IN-PATIENT BURDEN OF IBD: ANALYSIS OF 16,000 ADMISSIONS IN THE LOTHIAN IBD REGISTRY (LIBDR)}

Mathew Lyons*, Nikolas Plevris, Spyros Siakavellas, Laura Lucaciu, Philip Jenkinson, Ian Arnott, Charlie Lees, Gareth Jones. Edinburgh IBD Unit

\subsection{6/gutjpl-2020-bsgcampus. 199}

Introduction The Lothian IBD Registry (LIBDR) is a comprehensive record of all cases of IBD in Lothian, a geographical area in South East Scotland with a population of approximately 900,000. The current IBD prevalence in Lothian is $0.79 \%$ and predicted to reach $1 \%$ by 2028 . We aim to describe all-cause admission characteristics for IBD patients over the last 10 years and predict the consequences to healthcare provision as IBD prevalence continues to grow.

Methods The LIBDR was compiled through a capture-recapture derived prevalence estimation of IBD in Lothian ${ }^{1}$. Hospital admission rates for this cohort were derived using preexisting registries and electronic health record linkage by community health index (CHI) number, a unique identifier covering $100 \%$ of the population, between $01 / 01 / 2010$ (start of electronic recording of data) and 30/09/2019. All admissions $<24$ hour duration were excluded. All diagnosis codes were recorded using the ICD-10 system. Primary care prescription data was recorded using British National Formulary (BNF) codes. Clinic attendance data was available from 01/01/2015.

Results There were 17,836 hospital admissions for 3,400 of the 8,255 patients in the LIBDR prevalent cohort in the study period. Overall the number of admissions per prevalent case is decreasing over time (figure $1(\mathrm{a})$ ). IBD was the primary reason for admission in 3,715 of those admissions for 1,775 patients. Median length of stay for all admissions was 4 nights (IQR 2-9). Admissions for the cohort accounted for 202,857 beddays, an average of 23,000 per year. The commonest reason for admissions was infection (4,639 admissions for 2,064 patients, $15 \%$ of admissions), followed by Crohn's disease (K50.*,11.3\% of admissions) and ulcerative colitis (K51. *9.5\%). Of the patients admitted for infection, 88 patients required 197 ICU admissions. A primary care prescription for an antimicrobial, steroid, opioid or cytotoxic drug was given before 116 of these ICU admissions.

Less than half of patients admitted after 01/012015 were seen in clinic within 180 days prior to admission (400/870 patients). Despite this, the number of appointments per

Abstract P124 Figure 1 Change in (a) admissions and (b) appointments per prevalent case over time 